\title{
Integrated Behavioral Health in Primary Care for Adults with Complex Childhood Onset Medical and Developmental Diagnoses
}

\author{
Kristen E. Holderle ${ }^{1}\left[\right.$ D Ellen Poleshuck ${ }^{2} \cdot$ Tziporah Rosenberg $^{3} \cdot$ Tiffany Pulcino $^{4}$
}

Accepted: 15 June 2021 / Published online: 29 June 2021

(c) The Author(s), under exclusive licence to Springer Science+Business Media, LLC, part of Springer Nature 2021

\begin{abstract}
Increased life expectancy for individuals with complex pediatric-onset conditions means most of this population survive into adulthood. While this is great news for individuals and their families, the traditional adult medical model must adapt to extend the care provided by specialty pediatric practices to primary care. In this paper, we introduce a model of integrated behavioral health (IBH) in a primary care practice for adults with childhood onset medical and developmental conditions. Our discussion includes the role of IBH providers (i.e., psychologists, psychiatrists, and social workers) as members of the integrated team, patient engagement and response to treatment, and innovative ways we strive to meet patient needs. Our review of electronic health records of patients seen at the UR Medicine Complex Care Center suggest that IBH is feasible and highly utilized, with 216 patients (40\%) having had contact with an IBH provider on the team at least once. We discuss the challenges of meeting the longer-term needs of this complex patient population and our directions for future growth including creating peer and caregiver support networks, expanding services offered, and continued collaboration with community partners.
\end{abstract}

Keywords Integrated behavioral health $\cdot$ Multidisciplinary care $\cdot$ Pediatric-onset conditions $\cdot$ Sickle cell disease $\cdot$ Cystic fibrosis $\cdot$ Intellectual and developmental disabilities

\section{Introduction}

Advances in medicine have led to increased life expectancy for individuals with complex pediatric-onset conditions such as cystic fibrosis $(\mathrm{CF})$, intellectual and developmental disabilities (IDD), and sickle cell disease (SCD; Global Burden of Disease and Injury Incidence and Prevention Collaborators, 2017). More than $90 \%$ of this population are surviving into adulthood (Pai \& Schwartz, 2011). As these individuals age out of intensive specialty pediatric care, their transition

Kristen E. Holderle

Kristen_Holderle@urmc.rochester.edu

1 Department of Psychiatry and Pediatrics, University of Rochester Medical Center, 905 Culver Road, Rochester, NY 14609, USA

2 Department of Psychiatry and Obstetrics/Gynecology, University of Rochester Medical Center, Rochester, NY, USA

3 Department of Psychiatry and Family Medicine, University of Rochester Medical Center, Rochester, NY, USA

4 Department of Internal Medicine and Pediatrics, University of Rochester Medical Center, Rochester, NY, USA to adult care is associated with an increase in high-cost health care resource utilization (i.e., emergency room and inpatient care), deteriorating medical outcomes, decreased family satisfaction, and increased morbidity/mortality (Annunziato et al., 2007; Bonnie et al., 2015; Cole et al., 2015; Pai \& Ostendorf, 2011). In the US, there are more adults with complex pediatric-onset conditions such as CF, IDD, and SCD than there are children with the same. While specific illness groups experience unique challenges for patients transitioning to adult care, common barriers across chronic illness groups include relationships with providers, belief/expectations, skills/efficacy, knowledge, and access/ insurance (Gray et al., 2018).

Previous research has demonstrated a high comorbidity between physical and mental illnesses (Druss \& Walker, 2011; Egede et al., 2002; Goodwin et al., 2009; Reichard et al., 2011). Sartorious (2013) suggested that the simultaneous occurrence of physical and mental illnesses should be considered the rule rather than an exception. Studies of patients with CF have found higher rates of depression when compared with controls, with rates ranging from 29 to $46 \%$ (Quittner et al., 2008). Due to the impact of depression 
on treatment compliance and health related quality of life, the US CF Foundation recommends annual depression and anxiety screening for all individuals with $\mathrm{CF}$ age 12 and over (Quittner et al., 2016). Quittner and colleagues also recommend that patients with $\mathrm{CF}$ have access to behavioral interventions for distress associated with medical procedures as well as cognitive-behavioral and interpersonal psychotherapies for depression and anxiety.

Likewise, rates of depression in individuals with SCD range from 18 to $44 \%$ (Levenson, 2008) and are associated with increased pain and reduced quality of life. The Pain in Sickle Cell Epidemiological Study (PiCES) found that $27.6 \%$ of adults with SCD were depressed and $6.5 \%$ had an anxiety disorder and that depression and anxiety were related to self-reported pain, health-related quality of life, and opioid use (Levenson et al., 2008). Anxious patients also were more likely to meet their health care needs through frequent trips to the emergency room (Levenson et al., 2008). Socioeconomic status and cultural factors often make it difficult for patients with SCD to access behavioral health services, which can further exacerbate health-related challenges and increase utilization of emergency medical services, underscoring the importance of improving access to care for this population.

The Diagnostic Manual-Intellectual Disability2nd Edition (DM-ID-2) notes that individuals with IDD exhibit psychiatric and behavioral co-morbidity at rates that are two to three times greater than the general population. Furthermore, the general consensus among professionals is that $30-35 \%$ of people with IDD will experience a psychiatric disorder (APA, 2013). In general, there is a paucity of evidence-based behavioral health services for children or adults with IDD. While children benefit from early intervention, specialized pediatric care, and educational-related support services, as they age out of the educational and pediatric health systems, limited access to services in the adult health care arena leads to increased challenges. In addition, there are fewer opportunities for socialization and treatment of mental or behavioral disorders. Specific challenges to accessing health care for individuals with IDD include lack of adult providers with formal training, comfort, and time to adequately assess and treat young adults with IDD. This can be particularly challenging for those with comorbid mental health diagnoses given limited availably of behavioral supports and psychiatric services (Kim et al., 2017).

The high rate of depression, anxiety, and other comorbid psychiatric conditions in individuals with pediatric-onset illnesses and developmental disabilities impact multiple factors including overall health, well-being, pain, and quality of life. The Faces of Medicaid III Survey concluded that the high prevalence of psychiatric diagnoses among people with chronic medical conditions is an impetus for prioritizing improved integration of behavioral and medical care
(Kronick et al., 2009). Delivering behavioral and mental health care along with medical care in a primary care setting has increasingly been embraced by health care organizations. This integrated model of care differs from traditional mental health care in its approach to assessment, treatment, and collaboration within the health care team (Kelly \& Coons, 2012). Likewise, service delivery is often briefer than traditional mental health clinics both with regard to number and length of sessions (Funderburk et al., 2018). Multiple models have been developed for behavioral medicine concerns with evidence for its success for many difficulties, including insomnia/sleep hygiene, pain, and sexual health (Funderburk et al., 2018).

For vulnerable or chronically ill populations such as those with $\mathrm{CF}, \mathrm{SCD}$, and IDD, access to behavioral and mental health care within their primary care office reduces barriers to care and has the potential to increase service utilization and enhance treatment outcomes. Having all providers in the same physical location also addresses historically poor communication between mental health and medical systems, which has been associated with poor quality of care for people with comorbid conditions (Institute of Medicine, 2006). In this paper, we discuss the successes and challenges of implementing a model for IBH for a complex and high-needs patient population of adults $(19+)$ with pediatric-onset conditions. To our knowledge, our clinic is the first multidisciplinary primary care clinic for individuals with complex pediatric-onset condition to include IBH services as part of its multidisciplinary team.

\section{The UR Medicine Complex Care Center (CCC)}

The CCC opened in March 2016 with the goal of improving overall access to care for individuals aged $19+$ with pediatric-onset conditions. As of October 2018, the CCC patient panel included 537 patients with the following childhood onset diagnoses: IDD (280; 52\%), Cystic Fibrosis (13; 25\%), Sickle Cell Disease (65; 12\%), Type I Diabetes Mellitus (10; $2 \%)$, Spinal Cord Injury (10; 2\%), Genetic Syndrome (10; $2 \%)$, and $\operatorname{Other}^{1}(27 ; 5 \%)$.

Integral to this model is the presence of a comprehensive multidisciplinary care team including primary care, behavioral health care, care management, dentistry, nutrition management, and other therapies. In addition to the IBH team, discussed in greater detail below, during the time period reviewed in this manuscript, the CCC team included five physicians ( $0.5 \mathrm{cFTE}$ each), one nurse practitioner (1.0 FTE), three nurses (1.0 FTE each), an occupational therapist

\footnotetext{
${ }^{1}$ Includes childhood stroke, irritable bowel disease, traumatic brain injury, congenital heart disease, and muscular dystrophy.
} 
Table 1 Integrated behavioral health team members

\begin{tabular}{ll}
\hline Team members (FTE) & Roles and contributions \\
\hline Licensed Clinical Social Worker (1.0 FTE) & - Clinic Mental Health Coordinator, oversees annual mental health screening \\
& - Social worker on CF grant, provides annual screening and participates in advanced care planning \\
& grant \\
& - Liaison between clinic patients, providers, and community groups \\
& - Conducts annual mental health assessment \\
& - Provides evaluation, consultation, crisis management \\
& - Provides psychotherapy \\
& - Research and quality improvement initiatives \\
& - Individual psychotherapy, with a specialty focused on patients with medical illnesses \\
& - Administrative duties including overseeing IBH team \\
& - Research, quality improvement, and program development initiatives \\
& - Provide bereavement and wellness initiatives \\
& - Individual psychotherapy with focus on IDD population \\
& - Assessments for autism, intellectual disability, and adaptive skills \\
& - Ongoing research, program development, and quality improvement initiatives \\
& - Consultation and evaluation of patient medication for patients on complex medication regimens \\
& (e.g., multiple antipsychotics) \\
Clinical Psychologist 2 (0.4 FTE) & Provides recommendations to PCP who prescribes/manages medication
\end{tabular}

(OT; 0.2 FTE), a physical therapist (PT; 0.2 FTE), a registered dietician (RD; 1.0 FTE), and a pharmacist (1.0 FTE). CCC support staff includes a director of workforce development, a director of grants administration, practice manager, a care management team, and office support staff (including front desk personnel and referral support specialist). A co-located dental clinic includes multiple dentists, dental hygienists, a dental coordinator, and support personnel.

\section{The CCC Model of Integrated Behavioral Health}

The model used at the CCC was part of a larger hospitalwide initiative to deliver IBH services in primary care clinics to reduce emergency department visits. The goal was to support primary care practices utilizing a population-health approach for meeting patient behavioral healthcare needs through universal screening and the provision of short-term, evidence-based psychotherapy treatments, psychiatric consultation, and immediate BH access (e.g. warm-hand-offs). Under this model, patients are referred by their primary care provider (MD or NP) who identify the need for treatment through discussion with patient and/or based on results of mental health screening tools administered to all patients as part of their standard primary care. Our goals were to reduce barriers to care, improve overall patient care, and enhance provider communication and care coordination by utilizing a team-based integrated approach within the primary care setting. This is especially important for this medically fragile population who often have additional barriers to attending IBH appointments such as reduced physical mobility, frequent inpatient hospitalizations, disruption in care due to pain crises or other symptom exacerbations, and insurancerelated barriers.
Initially, the CCC IBH team consisted of one full-time licensed clinical social worker. As the need for services grew, the team expanded to include two part-time licensed psychologists and a consulting psychiatrist. The addition of licensed psychologists allowed for standardized assessment of intellectual disabilities, autism spectrum disorders, and adaptive skills to assist patients in obtaining needed supports and care. While our physicians and nurse practitioner are generally comfortable managing psychiatric medications, many of our IDD patients are prescribed multiple antipsychotics which may require additional expertise to manage. In addition, the complex medical needs of our patients is often challenging due to the need for complex regimens with potential interactions or side effects. Adding a consulting psychiatrist to the team allows these patients to be evaluated by a specialist who can make recommendations to the primary care provider without them having to be referred to a separate clinic or have long wait-times for an appointment. In addition, as we have a number of patients who have or will receive organ transplantation, our psychiatrist can conduct pre- and post-transplant evaluations to address potential risks.

The varied and specific roles and duties of each IBH provider are outlined in Table 1 . Services provided by the IBH team include support for practice-wide depression, anxiety, and substance use screening initiatives, standardized assessments of cognitive, developmental, and adaptive skills, evaluation and short-term treatment for psychiatric or behavioral concerns, psychoeducational support to patients and family members, and referral to longer-term services/community agencies as needed.

In addition to providing direct patient care, IBH providers support other CCC providers and staff through case 
consultation (including informal "curb side" consultations and during interdisciplinary rounds), professional development, and general support for working with this challenging population. These have included formal presentations on topics such as mental health screening, handling crises within the office setting, integrating the use of sensory tools with patients, and addressing grief. Informally, IBH providers are available to talk with providers who have questions about how to approach a specific concern with a patient. Other initiatives have included addressing provider wellness and bereavement related concerns, discussed in greater detail below.

IBH providers at the CCC are integrated as part of the team in a number of ways designed to enhance communication among providers and improve overall patient care. Clinic huddle is held $30 \mathrm{~min}$ before each clinic (i.e., 8-8:30 a.m. and 1-1:30 p.m. daily). During this multidisciplinary meeting, providers review the clinic schedule, discussing pertinent patient information including identifying those patients who may need to check-in with an IBH provider that day. IBH provider schedules are organized in a way that allows discrete appointment times for initial (i.e., new patient) and follow-up appointments with planned schedule gaps for warm hand-offs, curb-side consultations, and coordination with other provides. Similar to other IBH models, appointments are generally briefer than traditional psychotherapy (i.e., $30 \mathrm{~min}$ for follow-up) with a focus on specific, goal-oriented, evidence-based episodes of care. During their clinic times, when they are not seeing scheduled patients, IBH providers join other providers in the clinic charting room. This allows for cross-discipline consultation and for IBH providers to be available to meet with patients who may have an acute need (i.e., safety assessment or need for referral for substance use evaluation).

\section{Mental Health Screening and Assessment}

At the CCC we use population based screening via iPad to help identify individuals at risk for anxiety, depression, and substance use disorders. Patients without IDD are screened for depression using the Patient Health Questionnaire (PHQ; Kroenke et al., 2001). The PHQ-2 is administered using an algorithm which expands to the PHQ-9 based on positive responses to either question. In instances where paper measures are given (i.e., the iPads are not functioning or otherwise unavailable) we use the PHQ-9.

Given that approximately half of our patients have IDD and may not be able to complete standard depression screening forms, where appropriate, we use the Glasgow Depression Scale for People with a Learning Disability (GSD-LD; Cuthill et al., 2003). Our population-based screening also includes the Generalized Anxiety Disorder-7 item (GAD7) Scale (Spitzer et al., 2006; Swinson, 2006) for anxiety, the Drug Abuse Screening Test (DAST-10; Skinner, 1982) for substance use, and the AUDIT Alcohol Consumption Questions (AUDIT-C; Bush et al., 1998) for alcohol use.

As part of her role on a grant from the CF Foundation, the mental health coordinator conducts an annual individualized mental health assessments for CF patients. In addition to the administering the screening measures described above, this annual assessment includes a clinical interview to gather information on patient functional and communication status, advanced directives, health literacy, barriers to medical treatment, a thorough psychosocial assessment, history of mental health treatment, mental status exam, general coping, and treatment recommendations. This information is compiled into a report along with patient scores on the screening measures outline above and saved in the patient electronic medical record. As with all IBH notes, all members of the $\mathrm{CCC}$ team are able to access this document (i.e., there is no extra security or need to "break the glass" as in traditional mental health settings). After an initial pilot of using this screening with patients with $\mathrm{CF}$, it has been expanded for use with our entire clinic population.

Patients who are identified as needing behavioral health treatment (e.g., through screening cut off scores, provider assessment, or patient request for services) are referred to a member of the IBH team for an initial evaluation. An advantage of the IBH model is the opportunity for providers to introduce themselves to the patient during a medical appointment prior to their initial evaluation. In instances where screenings reveal safety concerns such as active suicidal ideation, we have workflows to conduct a safety assessment and safety planning or facilitate transfer to a more appropriate level of care (i.e., psychiatric emergency room) as needed. For those patients who receive behavioral health services from a community provider, with patient consent our IBH providers can coordinate care with that provider, share concerns regarding screening results, or consult regarding specific aspects of treatment, especially related to comorbid medical conditions.

\section{Additional Services Offered by CCC IBH}

Below we briefly review several innovative services that we have implemented to meet the needs of our patient population.

\section{Caregiver Support Groups}

Approximately $17-28 \%$ of the adult population provides care to a family member or friend who is ill or disabled (Coughlin, 2010; Schulz \& Tompkins, 2010). Of these, about 7\% are middle-aged parents providing care for their adult children (U.S. Department of Health and Human Services, 1998). Furthermore, approximately $71 \%$ of individuals with IDD 
live with a family caregiver (Williamson \& Perkins, 2014). Research suggests that caregivers are at risk for caregiver burden, an all-encompassing term which describes the physical, environmental, and financial toll of providing care. Specifically, caregiving can be associated with depression, financial strain, loss of educational or career goals, and social isolation (Shultz \& Sherwood, 2009; Vasileiou, 2017). While much focus has been placed on caregivers of young children, veterans, or the elderly, there is a paucity of data on caregivers of young adult children. Nevertheless, this group provides care to their child or family member for a long period of time, likely placing them at increased risk for caregiver burnout and other caregiving challenges. Higher caregiver burden, in turn, may have negative consequences on patient well-being.

We created a series of caregiver support groups to support the families of CCC patients. Groups occurred for $60 \mathrm{~min}$ over the lunch hour on a monthly basis. They were facilitated by a licensed psychologist who provided a brief overview of a specific topic for part of the time, allowing time for open discussion among group members. Session topics included caregiver burden, coping with stress, anxiety, and depression, assisting loved ones with transitions, effective communication, use of behavioral strategies, and conflict resolution. In general, family member feedback provided to the group facilitator suggested that groups were well-received and that the support and connections they made were valuable.

\section{Telemedicine}

Given the barriers many of our patients have to attending regular therapy appointments (e.g., wheelchair bound, live far away from the clinic), we have utilized the opportunity to provide therapeutic services using the telemedicine infrastructure within our hospital system even prior to the onset of the more global use of telehealth during the COVID-19 pandemic. This includes an initial in-person evaluation session, completion of appropriate consent forms, and use of a HIPAA compliant system. In one instance, it was identified that a patient would benefit from evidenced based Cognitive Processing Therapy (CPT; Resick et al., 2017) for her PTSD, yet her distance from the clinic prevented her from attending weekly sessions in person. The opportunity to provide CPT via telemedicine allowed the patient access to this evidenced based therapy that she otherwise would not have had access to.

A primary concern of using telehealth with this population is whether patients can have privacy and safety in their home setting. While the technology used is HIPAA compliant, patients with nursing or home-health aides or those who live in group homes may have difficulty finding a private place to connect for the visits. Prior to referring for telehealth services, providers assess for safety and privacy concerns to ensure confidentiality can be safely maintained. The telehealth platform also requires patients have access to specific technology (e.g., smart phone, computer, or tablet) to connect via the telemedicine platform. In addition, some individuals who have tried telehealth have reported finding it difficult to develop a therapeutic alliance and prefer being seen in person without the barrier that technology can create. Additional challenges arise over insurance reimbursement of these services, which varies based on specific insurer contracts. In general, despite challenges we have found the use of telehealth to be a good adjunctive option for some patients, in addition to occasional in-person appointments for individuals who are in need of IBH support but unable to physically be present at the office due to limitations of distance or physical constraints. When the COVID-19 pandemic began in March 2020, our existing infrastructure for providing telemedicine to some of our patients allowed us to quickly pivot to provide $100 \%$ of our services via telemedicine to protect our highly vulnerable population and allow them continued access to behavioral health services.

\section{Inpatient Visitation and Consultation}

CCC patients are frequently hospitalized to address acute (i.e., sickle cell crisis) or preventive (i.e., CF tune-up) medical needs. During these hospitalizations, IBH providers are available to consult with the inpatient medical team around emotional or behavioral concerns that may arise. For patients who are in ongoing therapy, providers may visit them in the hospital to reinforce learned skills and provide a familiar face and some comfort during a stressful time. For patients who require hospitalization for psychiatric needs, IBH providers also are available for consultation and to assist with discharge planning and transitioning patients between services.

\section{Patient Utilization of CCC IBH Services ${ }^{2}$}

We conducted a record review to better understand patient utilization of IBH services. This was conducted in September 2018, when the clinic had been open for 30 months. Of the 537 patients who engaged in primary care at the CCC, $216(40 \%)$ had utilized BH services, as measured by having at least one consultation with a $\mathrm{BH}$ provider. Of those that were seen for ongoing therapy by the CCC psychologist $(n=68)$, the following information was available: average number of appointments $=10.6$ (range 1-37), with an attendance rate of $65 \%$. A review of the most recent administration

\footnotetext{
${ }^{2}$ Given that this project describes a clinical program and is not research, activities were exempted from the purview of the University of Rochester Research Subjects Review Board.
} 
of PHQ-2/9 screenings for this subset of patients indicated an average score of 5.67 (indicative of mild levels of depression), with a range from 0 to 23 . While we have not yet objectively surveyed patient satisfaction, anecdotally, patient response to IBH has been favorable. Patients often tell providers they feel comfortable in the integrated setting, they appreciate that their behavioral health provider is knowledgeable about their medical illness(es), and they like the ability to be able to coordinate appointments with multiple providers on the same day. Next steps include assessing patient and family satisfaction and patient outcomes more systematically.

\section{Case Study}

Here, we briefly describe an episode of care for a patient referred for IBH services at our clinic. To maintain confidentiality, we have changed or removed identifying information. "Sam" is a 21-year-old male with Duchene's muscular dystrophy and other associated medical comorbidities including obstructive sleep apnea. He is wheelchair bound and lives with family members who, along with aides, assist him with ADLs and transportation. He was prescribed Lexapro $10 \mathrm{mg}$ by his primary care provider due to feelings of depression and "feeling stuck." He had experienced a number of transitions in his life and was struggling due to progressive loss of functioning and anxiety related to future goals. The onset of these difficulties seemed to coincide with his transition out of school where he was in a more supportive environment. He had no prior history of mental health treatment. He was referred to the IBH team to address these concerns and was seen by the first author for eight psychotherapy sessions over a 6-month period in 2018.

Session 1, the intake session, focused on gathering background information and setting treatment goals. Prior to this session, Sam's referral was discussed as a team during the pre-clinic planning and the primary care provider provided background information about Sam and the reason for referral. During the session, Sam insightfully articulated the struggle he felt being "a man stuck in a kid's body." He demonstrated good insight when talking about his difficulty regulating and communicating his emotions, as well as his desire to improve his relationships with others and engage in meaningful activities. Treatment primarily utilized the principles of Acceptance and Commitment Therapy (ACT; Hayes et al., 1999). ACT is a considered a "third-wave" cognitive-behavioral intervention focused on improving psychological flexibility through the use of acceptance and mindfulness processes as well as commitment and behavior change processes. (Hayes et al., 2013). ACT has been shown to be an effective form of treatment for a wide range of psychological and medical conditions. As of May 2019, 304 randomized controlled trials of ACT have been published providing support for this treatment model (Hayes, 2019).

During the course of treatment, Sam's case was reviewed with the team at each pre-clinic planning session on days he was scheduled for his therapy appointments. In addition, progress notes were included in his medical chart without the usual "break-the glass" privacy concerns that exist in traditional mental health settings so that his primary care provider and other members of the team could review them as needed. When Sam was seen for medical appointments and his case was presented at pre-clinic planning, the IBH provider also could provide relevant information from his ongoing treatment for discussion with the team.

During the initial phase of treatment, Sam was provided psychoeducation about ACT principles. In-session and homework practice included developing psychological flexibility skills including acceptance, mindfulness, and defusion techniques (e.g., checking in with his thoughts and physical sensations, labeling thoughts, allowing feelings as they occur). In addition he learned organizational tools and engaged in goal-setting and committed action. By the $8^{\text {th }}$ session, he was reporting improved mood and relationships. He also had taken several steps towards engaging in meaningful activities, including significant progress on a goal that was very important to him. It was mutually decided to take a break in treatment. As part of termination, skills learned in therapy were reviewed and relapse prevention was discussed. Sam was informed that he could re-engage in treatment at any point if symptoms returned or new concerns developed. When this episode of care was completed, the provider handed care back to the primary care provider who had continued to prescribe medication during psychotherapy. Even after the episode of care ended, Sam's IBH provider checked in with him when he was seen at the clinic for follow-up medical appointments and continued to be involved in teambased discussions about Sam's care at pre-clinic planning for his medical appointments. Nine months after treatment, Sam continued to do well despite some additional medical concerns, and had not needed to return to treatment.

\section{Discussion}

As described above, our initial chart review indicated that, 2.5 years after opening, 40 percent of the patient population had utilized IBH services for consultation and/or treatment. In general, providers and patients have embraced the idea of working with IBH specialists and have reported to us that they appreciate the opportunity to see these providers in coordination with their primary care provider or other specialists. It is our sense that IBH providers have become 
valuable members of the multidisciplinary team and their expertise is appreciated and welcomed.

Furthermore, as described above, we have developed and implemented a number of programs including caregiver support groups, telehealth services, provider wellness initiatives, and population-wide comprehensive mental health assessments. As demonstrated in our case example, our providers are able to provide evidence-based interventions in a short-term, flexible model within a primary care setting collaborating closely with providers. This allows this highly vulnerable population access to services they likely would not otherwise have been able to obtain. Next steps are to assess the impact of our IBH services on overall service utilization (i.e., inpatient, outpatient, and emergency department), associated service-related expenses, quality of life, symptoms of anxiety and depression, and physical health outcomes. We would hope that the benefit of IBH services would be indicated through reduction in service utilization, especially emergency room visits, reduction in PHQ-2/9 and GAD-7 scores, improvement in quality of life, and increased engagement in behavioral health care for individuals who historically have had many barriers to accessing care.

We have encountered several challenges conducting IBH in this setting with this particular patient population. First, given the chronic terminal illnesses of many of our patients, there was a high number of patient deaths (approximately 25 ) within the first two years of opening the clinic. This has provided an opportunity for the IBH team to evaluate and address the needs of patients, families, and providers as it relates to palliative care, death and dying, and provider burden/burnout. We have done this in multiple ways. On a system-wide level, we have created workflows for disseminating information about patient deaths so that all team members receive this information. We also dedicate time once a month at our weekly huddle to allow members of the team to talk openly about topics related to bereavement and stress/burnout. During this time we pass around sympathy cards for the patient's family and/or group home or other caregivers and team members are invited to share their thoughts about the patient in writing. We memorialize patients who have died in a monthly ceremony where team members share stories about the patient and place their initials on a tree that decorates the clinic wall behind our nurse's station. As providers and staff may not always want to share their thoughts related to grief and burnout publically, one of the IBH team members (the first author) is available to meet with team members individually upon request. Finally, we have included behavioral health providers into advanced care planning meetings and creating quality improvement projects to address provider stress and burnout.

Secondly, our IBH model is designed for short-term episodes of care focused on evidence-based treatments for specific goals, utilizing a model of brief ( $30 \mathrm{~min})$ appointments rather than the traditional 60-min therapy hour. Ideally, patients who need longer-term mental health care would be referred to our hospital system's outpatient ambulatory clinic or community agencies. In reality, we have found that our complex patient population often requires longer-term care and has difficulty accessing it for a number of reasons. Individuals who are wheelchair-bound or have complex medical needs, for example, have had difficulty finding providers or clinics where these needs can be managed appropriately with providers who understand their comorbid medical illnesses. Also, due to IDD, physical challenges, and the participation of caregivers in treatment, many of our patients need a full 60 min to meet their care needs.

Likewise, over half of CCC patients have an IDD. Nationwide, there is a general lack of evidence-based treatment for individuals with IDD, and, within our community we have struggled to find outpatient providers or service agencies to meet the needs of our transition-age and adult patients, especially those with dual diagnoses. Thus, we often have to implement our model flexibly and follow a more traditional psychotherapy model (i.e., longer session lengths and treatment duration) for patients with IDD as there are fewer options for them. Patients who have required crisis-oriented mental health services (i.e., emergency room visits, use of community crisis services, or psychiatric hospitalization) have encountered similar barriers including lack of ability to provide for medical needs during hospitalization, lack of familiarity with developmental disabilities, communication barriers for non-verbal patients, and lack of understanding or support around dual diagnoses.

Additional challenges have included coordinating care between multiple systems and caregivers. Many of our patients live in group homes where they are cared for by group home staff in addition to engaging on a regular basis with staff from day programs, other community organizations, and other caregivers (including parents, guardians, and other family members). This can be challenging when implementing treatment plans, especially around environmental or behavioral interventions, as it takes coordinated efforts to ensure all parties are working together and maintaining consistency. While we attempt to include caregivers in the treatment process where appropriate, we also rely on our team of care managers to help us bridge gaps with staff and supports from group homes, day programs, and community agencies. We recently hired a medical social worker to help fill needs in these areas.

\section{Summary and Future Directions}

During the first few years our clinic has existed, we have identified a number of needs we are working to address. First, consistent with previous research (see Vasileiou 
et al., 2017), our patients and their caregivers self-report to providers that they feel isolated. The creation and implementation of caregiver groups was one way we sought to provide education and support to parents/caregivers. In addition, our patients also would benefit from groups that can address issues related to peer support, especially around specific medical illnesses, and transition/social skills related concerns for young adults with high-functioning autism spectrum disorder. We have especially noted a cohort of young adult males who struggle with regard to establishing romantic relationships and increased independence (financial, housing, etc.). Our primary barrier to implementing additional educational and support programming has been limited workforce hours, so we hope to involve psychology or social work trainees to help us create better programming for our patients and their families.

Many of our patients experience chronic pain which impacts their functioning and overall quality of life. Many are prescribed chronic opioid medications. We are in the process of starting evidence-based pain management groups for individuals with SCD. In addition, we plan to offer evidence based individual treatment including the use of biofeedback to address these concerns. The behavioral health team also is involved in the creation and implementation of a practice-wide opioid use screening measure and pain policy.

As mentioned above, we have a co-located dental clinic which services our patient population. We hope to expand our behavioral health services to include collaboration with dental staff to improve patient overall treatment compliance and oral health.

Finally, we hope to use the expertise of our IBH team to train other behavioral health providers in our community about the unique challenges of working with our patient population. Our goal is to do this through formal training, consulting with community providers regarding best practices, and creating supervised training opportunities within our clinic for behavioral health trainees (i.e., psychology interns, psychology post-doctoral fellows, social work interns, and psychiatry residents). Currently, two members of our IBH team are contributing to an Autism ECHO for mental health, an 8-session video conference designed to create a knowledge network among mental health providers around autism spectrum disorders. In addition, we have developed a practicum for psychology graduate students interested in gaining experiences working with our patient population. This training program includes heath psychology and IDD tracks. We also provide shadowing opportunities for psychology interns and postdoctoral fellows who are interested in learning more about IBH with our patient population and are investigating funding opportunities for a formal training program.

Since opening in 2016, the CCC has experienced growth in a number of areas. We have expanded the age group we serve to include patients 16 and older. This allows us meet a need identified in our community and continue to assist with transtion from adolescent specialty services to adult primary care. Likewise, our current primary care patient panel has grown to greater than 900 patients. To meet the expanding IBH needs, we have enrolled as a site in the New York State Collaborative Care Medicaid Program (CCMP). This allows us to be reimbursed for flexible, non-traditional behavioral health interventions (e.g., phone calls, service provided as part of the medical visits). Eligible patients are identified and enrolled based on depression and/or anxiety screening, the annual mental health assessment, or referral from their medical provider. We hope this allows us to better serve the portion of our population that has had difficulty attending appointments outside of medical visits, those who have struggled to engage within our current IBH model, and/or those lost to contact. The addition of the CCMP to our IBH services will increase our ability to be flexible, comprehensive, and integrative in providing behavioral health services to those who need them. We also have added Applied Behavior Analaysis (ABA) providers to our team to provide $\mathrm{ABA}$ for those in our IDD population that would benefit from these services.

IBH team members have become an integral part of the multidiscplinary team at the CCC. They are often sought out not only for patient related consultation and concerns and training opportunitites, but as collaborators on research and academic projects. IBH services have been well-received by the patient population. We continue to strive for flexible, integrated, and innovative ways to meet the unique needs and challenges of our vulnerable patient population.

Funding None.

\section{Declarations}

Conflict of interest Kristen E. Holderle, Ellen Poleshuck, Tziporah Rosenberg, and Tiffany L. Pulcino declare that they have no conflict of interest.

Human and Animal Rights This paper does not contain data from studies with human participants or animals performed by any of the authors.

Informed consent We were providing information about program development and not enrolling people in a study that involved informed consent.

\section{References}

American Psychiatric Association. (2013). Diagnostic and statistical manual of mental disorders, (5th ed.). https://doi.org/10.1176/ appi.books. 9780890425596

Annunziato, R. A., Emre, S., Shneider, B., Barton, C., Dugan, C. A., \& Shemesh, E. (2007). Adherence and medical outcomes in pediatric 
liver transplant recipients who transition to adult services. Pediatric Transplant, 11, 608-614. https://doi.org/10.1111/j.1399-3046. 2007.00689.x

Bonnie, R. J., Stroud, C., Breiner, H, editors. Investing in the Health and Well-Being of Young Adults. Washington (DC): National Academies Press (US); (2015). Committee on Improving the Health, Safety, and Well-Being of Young Adults; Board on Children, Youth, and Families; Institute of Medicine; National Research Council; Available from: https://www.ncbi.nlm.nih.gov/ books/NBK284787/. https://doi.org/10.17226/18869

Bush, K., Kivlahan, D. R., Mcdonell, M. B., Fihn, S. D., \& Bradley, K. A. (1998). The AUDIT alcohol consumption questions (AUDITC): An effective brief screening test for problem drinking. Ambulatory Care Quality Improvement Project (ACQUIP). Alcohol Use Disorders Identification Test. Archives of Intermal Medicine, 158, 1789-1795. https://doi.org/10.1001/archinte.158.16.1789

Cole, R., Ashok, D., Razack, A., Azaz, A., \& Sebastian, S. (2015). Evaluation of outcomes in adolescent Inflammatory Bowel Disease patients following transfer from pediatric to adult health care services: Case for transition. Journal of Adolescent Health, 57, 212-217. https://doi.org/10.1016/j.jadohealth.2015.04.012

Coughlin, J. (2010). Estimating the impact of caregiving and employment on well-being. Outcomes \& Insight in Health Management, 2(1), 1-7.

Cuthill, F. M., Espie, C., \& Cooper, S.-A. (2003). Development and psychometric properties of the Glasgow Depression Scale for people with a learning disability: Individual and carer supplement versions. The British Journal of Psychiatry, 182, 347-353. https:// doi.org/10.1192/bjp.182.4.347

Druss, B. G., \& Walker, E. R. (February 2011). Mental disorders and medical comorbidity. Research synthesis report no. 21. Princeton, NJ: the Robert Wood Johnson Foundation.

Egede, L. E., Zheng, D., \& Simpson, K. (2002). Comorbid depression is associated with increased health care use and expenditures in individuals with diabetes. Diabetes Care, 25, 464-470. https:// doi.org/10.2337/diacare.25.3.464

Funderburk, J. S., Shepardson, R. L., Wray, J., Acker, J., Beehler, G. P., Possemato, K., Wray, L. O., \& Maisto, S. A. (2018). Behavioral medicine interventions for adult primary care settings: A review. Family, Systems, and Health, 36 (3), 368-399. https://doi.org/10. 1037/fsh0000333

Global Burden of Disease and Injury Incidence and Prevalence Collaborators. (2017). Global, regional, and national incidence, prevalence, and years lived with disability for 354 diseases and injuries for 195 countries and territories, 1990-2017: A systematic analysis for the global burden of disease study 2017. The Lancet, 392, 1789-1858. https://doi.org/10.1016/S0140-6736(18)32279-7

Goodwin, R. D., Davidson, K. W., \& Keyes, K. (2009). Mental disorders and cardiovascular disease among adults in the United States. Journal of Psychiatric Research, 43, 239-246. https://doi.org/10. 1016/j.jpsychires.2008.05.006

Gray, N., Schaefer, M. R., Resmini-Rawlinson, A., \& Wagoner, S. T. (2018). Barriers to transition from pediatric to adult care: A systematic review. Journal of Pediatric Psychology, 43, 488-502. https://doi.org/10.1093/jpepsy/jsx142

Hayes, S. C. (2019 January). Contextual science: The state of the evidence. https://contextualscience.org/state_of_the_act_evidence

Hayes, S. C., Levin, M. E., Plumb-Vilardaga, J., Villatte, J. L., \& Pistorello, J. (2013). Acceptance and commitment therapy and contextual behavioral science: Examining the progress of a distinct model of behavioral and cognitive therapy. Behavior Therapy, 44, 180-198. https://doi.org/10.1016/j.beth.2009.08.002

Hayes, S. C., Strosahl, K., \& Wilson, K. (1999). G. An experiential approach to behavior change. Guilford Press.

Institute of Medicine (U.S.). (2006). Committee on crossing the quality chasm. Adaptation to mental health and addictive disorders. improving the quality of health care for mental and substance-use conditions. Washington.

Kelly, J. F. \& Coons, H. L. (2012). Integrated health care and professional psychology: Is the setting right for you? Professional Psychology: Research and Practice, 43(6), 586-595. https://doi. org/10.1037/a0030090

Kim, R., Steinwhy, C., Gabel, J., \& Jan, S. (2017). Transitioning to adulthood: Unique health care challenges for youth with intellectual/developmental disabilities. Policylab Policy Brief, Spring 2017.

Kroenke, K., Spitzer, R. L., \& Williams, J. B. W. (2001). The PHQ-9: Validity of a brief depression severity measure. Journal of General Internal Medicine, 16(9), 606-613. https://doi.org/10.1046/j. 1525-1497.2001.016009606.x

Kronick R. G., Bella M., \& Gilmer T. P. (2009). The faces of medicaid III: Refining the portrait of people with multiple chronic conditions. Center for Health Care Strategies, Inc.

Levenson, J. L., McClish, D. K., Dahman, B. A., et al. (2008). Depression and anxiety in adults with sickle cell disease: The PiSCES project. Psychosomatic Medicine, 70(2), 192196. https://doi.org/ 10.1097/PSY.0b013e31815ff5c5

Levenson, J. L. (2008). Psychiatric issues in adults with sickle cell disease. Primary Psychiatry, 15(5), 45-49.

Pai, A. L. H., \& Ostendorf, H. M. (2011). Treatment adherence in adolescents and young adults affected by chronic illness during the health care transition from pediatric to adult health care: A literature review. Childrens Health Care, 40, 16-33. https://doi. org/10.1080/02739615.2011.537934

Pai, A. L. H., \& Schwartz, L. A. (2011). Introduction to the special section: Health care transitions of adolescents and young adults with pediatric chronic conditions. Journal of Pediatric Psychology, 36 , 129-133. https://doi.org/10.1093/jpepsy/jsq100

Quittner, A. L., Barker, D. H., Snell, C., Grimley, M. E., Marciel, K., \& Cruz, I. (2008). Prevalence and impact of depression in cystic fibrosis. Current Opinion in Pulmonary Medicine, 14, 582-588. https://doi.org/10.1097/MCP.0b013e3283121cf1

Quittner, A. L., Abbott, J., Georgiopoulos, A. M., et al. (2016). International committee on mental health in cystic fibrosis: Cystic Fibrosis Foundation and European Cystic Fibrosis Society consensus statements for screening and treating depression and anxiety. Thorax, 71, 26-34. https://doi.org/10.1136/thoraxjnl-2015-207488

Reichard, A., Stolzle, H., \& Fox, M. H. (2011). Health disparities among adults with physical disabilities or cognitive limitations compared to individuals with no disabilities in the United States. Journal of Disability Health, 4, 59-67. https://doi.org/10.1016/j. dhjo.2010.05.003

Resick, P. A., Monson, C. M. \& Chard, K. M. (2017). Cognitive processing therapy for PTSD: A Comprehensive Manual. Guilford Press.

Sartorious, N. (2013). Comorbidity of mental and physical diseases: A main challenge for medicine of the 21 st century. Shanghai Archives of Psychiatry, 25, 68-69. https://doi.org/10.3969/j.issn. 1002-0829.2013.02.002

Schulz, R., \& Tompkins, C. A. (2010). Informal caregivers in the United States: Prevalence, caregiver characteristics, and ability to provide care. In the role of human factors in home health care (pp. 117-143). Washington, DC: The National Academies of Sciences Press.

Shultz, R., \& Sherwood, P. R. (2005). Physical and mental health effects of family caregiving. American Journal of Nursing, 108, 23-27. https://doi.org/10.1097/01.NAJ.0000336406.45248.4c

Skinner, H. A. (1982). The drug abuse screening test. Addictive Behavior, 7, 363-371.

Spitzer, R. L., Kroenke, K., Williams, J. B. W., \& Löwe, B. (2006). A brief measure for assessing generalized anxiety disorder: The 
GAD-7. Archives of Internal Medicine, 166, 1092-1097. https:// doi.org/10.1001/archinte.166.10.1092

Swinson, R. P. (2006). The GAD-7 scale was accurate for diagnosing generalised anxiety disorder. Evidence-Based Medicine, 11, 184. https://doi.org/10.1136/ebm.11.6.184

U. S. Department of Health and Human Services (1998). Informal Caregiving: Compassion in Aging, 1-28.

Vasileiou, K., Barnett, J., Barreto, M., Vines, J., Atkinson, M., Lawson, S., \& Wilson, M. (2017). Experiences of loneliness associated with being an informal caregiver: A qualitive investigation. Fronteirs in Psychology, 8, 1-11. https://doi.org/10.3389/fpsyg. 2017.00585
Williamson H. J., \& Perkins E. A. (2014). Family caregivers of adults with intellectual and developmental disabilities: outcomes associated with U.S. services and supports. Intellectual and Developmental Disabilities, 52(2), 147-159. doi: https://doi.org/10.1352/ 1934-9556-52.2.147

Publisher's Note Springer Nature remains neutral with regard to jurisdictional claims in published maps and institutional affiliations. 